\title{
GGS Prize 2008
}

The Editors and Advisory Board of Genes \& Genetic Systems are pleased to announce that the GGS Prize 2008 has been awarded to the authors for their paper:

\section{Mre11 mediates gene regulation in yeast spore development}

by Kazuto Kugou, Hiroyuki Sasanuma, Kouji Matsumoto, Katsuhiko Shirahige and Kunihiro Ohta, published in Genes \& Genetic Systems (2007) 82: 21-33.

This paper was selected by the editors and the members of the advisory board of Genes \& Genetic Systems as the best article published in Genes \& Genetic Systems in 2007.

Arguments supporting this decision were:

"Meiotic homologous recombination is a pivotal genetic event in sexually reproducing organisms. Initiation of meiotic recombination requires a lot of proteins including Mre11 and Spo11. Mre11 is a recombination enzyme that plays essential roles in DNA double strand break (DSB) repair, telomere maintenance, and formation of Spo11-mediated meiotic DSBs. The authors previously demonstrated that yeast Mre11 is involved in the meiotic changes of local chromatin configuration at recombination hot spots, most of which are located in promoter-containing intergenic regions. Loss of Mre11 confers deficiencies in the DSB formation and meiotic chromatin alteration at DSB sites. Using transcriptome analysis with DNA microarray, the authors indicated that mre11 mutants exhibit a specific loss of meiotic activation of a certain class of middle sporulation genes. Interestingly, most of these affected genes have the middle sporulation element (MSE) in their 5' upstream regions. Analysis of gene regulation using MSE-lacZ reporters revealed that the C-terminal region of Mre11, which is required for the meiotic chromatin transition at DSB sites, is essential in the chromatin changes at DSB sites. These results suggested that Mre11 is also important in meiotic gene regulation of a certain class of middle sporulation genes, as well as initiation of meiotic recombination. This paper for the fist time illustrates a new view of integrated regulation of meiotic recombination initiation and regulation of genes related to cell wall and spore formation." 